\title{
Standardized Uptake Value Peak
}

National Cancer Institute

\section{Source}

National Cancer Institute. Standardized Uptake Value Peak. NCI Thesaurus. Code C95013.

Standardized uptake value of the average for a limited number pixels (voxels) that includes the pixel (voxel) having SUVmax. 